\title{
Crescimento relativo do camarão canela Macrobrachium amazonicum (Heller) (Crustacea, Decapoda, Palaemonidae) em viveiros
}

\author{
Patrícia M.C. Moraes-Riodades ${ }^{1,2}$ \\ Wagner C. Valenti ${ }^{1,3}$
}

\begin{abstract}
Relative growth of Amazon river prawn Macrobrachium amazonicum (Heller) (Crustacea, Decapoda, Palaemonidae) in earthen ponds. Some morphometric relationships in Macrobrachium amazonicum (Heller, 1862) reared in earthen ponds were studied. A total of 239 individuals were collected, sexed and sorted to juvenile or adult. Total length (Lt), post-orbital length (Lpo), carapace length ( $\mathrm{Lcp}$ ) and queliped length (Lql) were measured. The relationships $\mathrm{Lt} / \mathrm{Lpo}, \mathrm{Lpo} / \mathrm{Lcp}$ and $\mathrm{Lt} / \mathrm{Lcp}$ are the same for juveniles, males and females, indicating unchanged growth pattern during post-larval ontogenetic development. While $\mathrm{Lt} / \mathrm{Lpo}$ showed isometric growth, Lpo/Lcp and Lt/Lcp showed negative allometry. On the other hand, for the Lq1/Lcp relationship, juveniles showed isometric growth, females slight positive allometry and males a strong positive allometry. It suggests that the importance of chelipeds may be different in these groups. Quelipeds play important role on food capture and on agonistic, social and reproductive behavior. Therefore, inter and intraspecific interactions may change during prawn growth, even after morphological maturation.
\end{abstract}

KEY WORDS. Crustacea, Macrobrachium amazonicum, freshwater prawn, relative growth, Amazon river prawn

Macrobrachium amazonicum (Heller, 1862), Palaemonidae, apresenta ampla distribuição geográfica, ocorrendo desde o Amapá até o Paraná (HoLTHUIS 1952; BIALETZKI et al. 1997). Tem importância ecológica e econômica, sendo largamente explorado pela pesca artesanal na região Nordeste (GURGEL \& MATOS 1984; NEw et al. 2000) e nos Estados do Pará e Amapá (ODINETZ-COLlaRT 1987; OdinETZ-Collart \& Moreira 1993; Moraes-Riodades et al. 1999). Além disso, é a espécie nativa do Brasil com maior potencial para a aqüicultura (KUTTY et al. 2000), sendo que cultivos em escala piloto vem sendo realizados no Pará (MORAES-RIODADES \& VALENTI 2001).

Os estudos referentes a $M$. amazonicum realizados até o presente têm se concentrado principalmente na ecologia e biologia pesqueira de populações naturais (ODinetZ-Collart 1987, 1991a, b; Odinetz-Collart \& MOREIRA 1993; MOREIRA \& ODINETZ-COLlaRT 1993; OdinETZ-COLlaRT \& MAGALHÃES 1994). Outros aspectos, tais como, a fecundidade (LOBÃO et al. 1986; SCAICO 1992; ODINETZ-COLLART \& RABELO 1996), o desenvolvimento gonadal (BRAGAGNOLI

1) Departamento de Biologia Aplicada, FCAV e Centro de Aqüicultura, Universidade Estadual Paulista. 14884-900 Jaboticabal, São Paulo, Brasil.

2) Bolsista de Doutorado da FAPESP. E-mail: riodades@caunesp.unesp.br

3) Bolsista de Produtividade em Pesquisa do CNPq. E-mail: valenti@ caunesp.unesp.br

Revta bras. Zool. 19 (4): 1169 - 1176, 2002 
\& GROTTA 1995), o desenvolvimento larval (GUEST 1979; ROMERO 1982; BARRETO \& SOARES 1982; MAGALHÃES 1985; LOBÃO et al. 1987; ROJAS et al. 1990), a alimentação e manutenção dos animais em laboratório (ALVES 1986; ROVERSO et al. 1990; LOBÃO et al. 1994) também foram investigados.

$\mathrm{O}$ estudo do crescimento relativo refere-se às relações entre dimensões do corpo ou de órgãos. É importante para o conhecimento das relações entre as várias partes do corpo, possibilitando a interconversão de dados de uma dimensão em outra e constitui-se em subsídio importante para a delimitação de populações e para vários estudos taxonômicos (HARTNOLL 1982; VALENTI et al. 1989). Além disso, pode fornecer subsídios para o reconhecimento de estágios de maturação morfológica (REIGADA \& NEGREIROS-FRANSOZO 1999). Estas relações são expressas por equações matemáticas e vem sendo bastante utilizadas para analisar o crescimento em crustáceos (HARTNOLL 1982).

Neste trabalho, foram determinadas as equações que relacionam o comprimento total ao comprimento pós-orbital e os comprimentos total, pós-orbital e do segundo quelípodo direito ao comprimento da carapaça, em M. amazonicum.

\section{MATERIAL E MÉTODOS}

Este trabalho foi realizado no Setor de Carcinicultura do Centro de Aqüicultura, Universidade Estadual Paulista (UNESP, CAUNESP), campus de Jaboticabal, São Paulo. No período de março a outubro de 2001, foram coletados e analisados 239 exemplares de $M$. amazonicum, sendo 52 jovens (sexo indeterminado), 72 fêmeas e 115 machos. Todos foram provenientes de dez viveiros de fundo natural, localizados na região nordeste do Estado de São Paulo (altitude de $595 \mathrm{~m}, 21^{\circ} 15$, 22 "S e $48^{\circ} 8^{\prime} 58^{\prime \prime} \mathrm{W}$ ). Estes foram estocados com pós-larvas produzidas no Laboratório de Larvicultura de Camarões do CAUNESP, a partir de reprodutores provenientes do nordeste do Pará, em densidades variando entre 10 e 20 indivíduos $/ \mathrm{m}^{2}$. Os camarões foram alimentados diariamente com ração balanceada, peletizada de conteúdo protéico de $32 \%$, na proporção de $5 \%$ da sua biomassa, fornecida no período da tarde. Foram analisados animais com idades variando entre um e vinte e quatro meses para contemplar todas as fases de desenvolvimento.

Os camarões coletados foram separados em juvenis com sexo indeterminado, machos e fêmeas. A seguir, foram medidos da extremidade do rostrum à extremidade do telson e, para cada grupo, considerou-se aproximadamente cinco animais de cada classe de comprimento (classes de $5 \mathrm{~mm}$ ). Isto é necessário porque, no estudo do crescimento relativo, é importante a obtenção de animais de todas as classes de tamanho, que devem ser igualmente representadas.

De cada exemplar, foram determinadas as seguintes dimensões (Fig. 1): 1) comprimento total (Lt): distância entre a margem distal do rostrum até a extremidade distal do telson; 2) comprimento pós-orbital total (Lpo): distância entre a margem posterior do olho direito até a extremidade distal do telson com o animal estendido; 3) comprimento da carapaça (Lcp): distância entre a margem posterior da órbita direita até o ponto médio da margem posterior da carapaça; 4) comprimento do segundo quelípodo direito (Lql): distância entre a margem proximal do ísquio até a extremidade distal do própodo. 


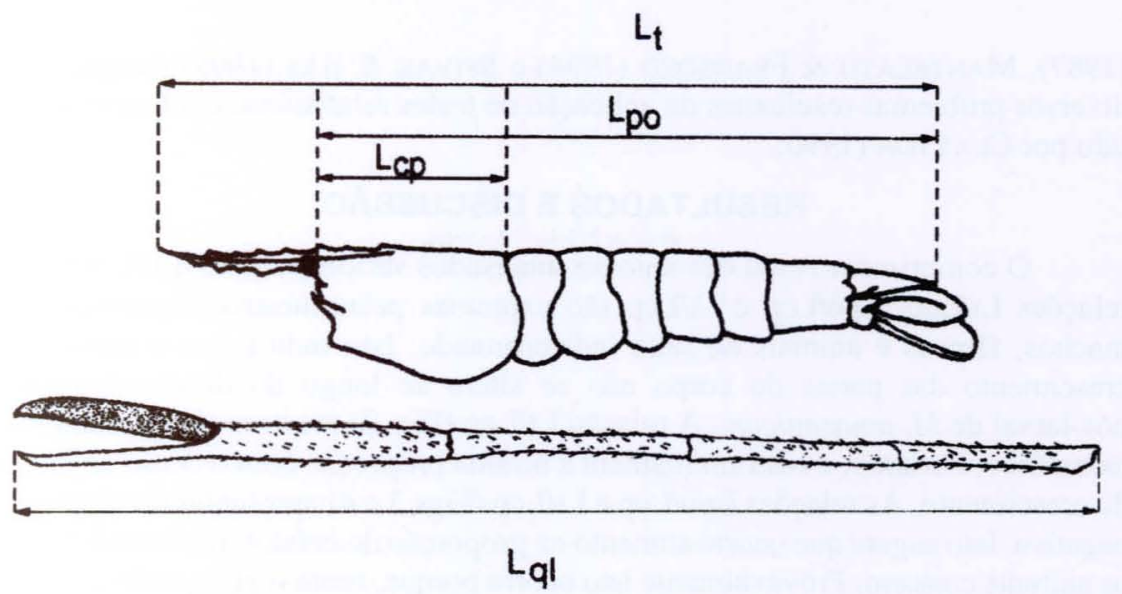

Fig. 1. Dimensões usadas na análise morfométrica. (Lt) comprimento total, (Lpo) comprimento pós-orbital total, (Lcp) comprimento da carapaça, (Lql) comprimento do segundo quelípodo direito.

O comprimento total dos animais adultos foi determinado por meio de um paquímetro de madeira com precisão $1 \mathrm{~mm}$. Todas as demais dimensões foram obtidas, utilizando-se um paquímetro digital com precisão $0,05 \mathrm{~mm}$.

Para o estudo do crescimento relativo, foi usada a equação alométrica $\mathbf{y}=\mathbf{a} \mathbf{x}^{\mathbf{b}}$ (HARTNOLL 1982) na forma $\operatorname{logarítmica,~ou~seja:~} \log \mathbf{y}=\log \mathbf{a}+\mathbf{b} \log \mathbf{x}$, sendo " $x$ " o tamanho do órgão de referência a partir do qual será estimado o tamanho do órgão "y"; "a" uma constante relacionada ao intercepto no eixo das ordenadas e "b" a constante de crescimento alométrico.

Nas relações morfométricas, as duas variáveis analisadas (x e y) apresentam variação aleatória e, portanto, obedecem ao Modelo II de regressão (SOKAL \& ROHLF 1995). Neste caso, o ajuste das equações pelo método dos Mínimos Quadrados, embora muito usado, fornece uma estimativa viesada da constante de crescimento alométrico (RICKER 1973). Portanto, no presente trabalho, os parâmetros da equação alométrica foram determinados pelo método do Eixo Maior Reduzido (RICKER 1973; SOKAL \& ROHLF 1995). Este método, também chamado Regressão da Média Geométrica ou Regressão Funcional é o mais adequado para estudos de crescimento relativo (HARTNOLL 1982; LOVETT \& FELDER 1989).

Segundo HARTNOLL (1982) e RoDRIGUES (1985), considera-se a alometria negativa, decrescente ou minorante, quando $b<1$, ou seja, o crescimento de um órgão, ou parte do corpo é mais lento em relação ao órgão de referência. Se b $>1$, a alometria é dita positiva, crescente ou majorante. Nesse caso, o órgão ou parte do corpo tem um crescimento mais rápido em relação ao outro de referência. Quando $\mathrm{b}=1$, chama-se isometria, pois os dois órgãos ou segmentos estão crescendo na mesma proporção. Considerou-se, no presente trabalho, alometria ṇegativa quando $\mathrm{b} \leq 0,90$, isometria quando $0,90<\mathrm{b}<1,10$ e alometria positiva quando $\mathrm{b} \geq 1,10$. Este procedimento vem sendo adotado por vários autores, tais como KURIS et al. 
(1987), MANTElato \& Fransozo (1994) e SPIVAK \& BAS (1999) porque evita diversos problemas resultantes da aplicação de testes estatísticos, conforme discutido por ClAYTON (1990).

\section{RESULTADOS E DISCUSSÃO}

O comprimento total dos animais analisados variou entre 15 e $132 \mathrm{~mm}$. As relações $\mathrm{Lt} / \mathrm{Lpo}$, Lpo/Lcp e Lt/Lcp são expressas pelas mesmas equações, nos machos, fêmeas e animais de sexo indeterminado. Isto indica que o padrão de crescimento das partes do corpo não se altera ao longo do desenvolvimento pós-larval de $M$. amazonicum. A relação Lt/Lpo (Fig. 2) mostrou um crescimento isométrico. Portanto, o rostrum mantém a mesma proporção com o corpo ao longo do crescimento. As relações Lpo/Lcp e Lt/Lcp (Figs 3 e 4) apresentaram alometria negativa. Isto sugere que ocorre aumento na proporção do cefalotórax à medida que os animais crescem. Provavelmente isto ocorre porque, nesta região, estão concentrados os órgãos vitais do camarão (coração, estômago, gônadas e brânquias). Espera-se que, com o desenvolvimento desses órgãos, o cefalotórax aumente proporcionalmente mais (KURIS et al. 1987). O crescimento na proporção do cefalotórax ao longo do desenvolvimento ontogenético também foi observado em M. rosenbergii (de Man, 1879) (RAO 1967). Por outro lado, em M. holthuisi Genofre \& Lobão, 1976 (LOBÃo \& LONA 1979), M. carcinus (Linnaeus, 1758) (LOBÃO et al. 1986) e M. acanthurus (Wiegmann, 1836) (VALENTI et al. 1989), o cefalotórax apresenta crescimento isométrico em relação ao comprimento do abdômen e ao comprimento total.

A relação Lql/Lcp (Fig. 5) é expressa por equações diferentes nos animais juvenis com sexo indeterminado, machos e fêmeas. A inflexão observada, provavelmente corresponde à muda puberal e marca o início da maturação morfológica. Esta ocorre quando os animais apresentam comprimento total ( $\mathrm{Lt}$ ) entre $50-55 \mathrm{~mm}$. Portanto, a partir desse tamanho, os camarões apresentam características sexuais secundárias de adulto. Esta fase pode corresponder ou não à maturidade funcional (REIGADA \& NEGREIROS-FRANSOZO 1999). Para elucidar esse aspecto, é necessário o estudo morfológico das gônadas. O crescimento do quelípodo é isométrico em relação à carapaça até a maturidade. A partir de então, observa-se alometria ligeiramente positiva nas fêmeas e altamente positiva nos machos. Isto indica que os quelípodos apresentam importância diferenciada em animais jovens, fêmeas e machos.

Os quelípodos são apêndices de grande importância para os camarões de água doce. Estes tem função na coleta de alimentos, na ocupação e defesa do território, nos comportamentos agonísticos em geral, na manutenção da estrutura social, no comportamento de corte e durante a cópula (NAGAMINE \& KNIGHT 1980; VOLPATO \& HOSHINO 1984; VALENTI 1985, 1987; KARPLUS et al. 1992; ISMAEL \& NEW 2000). Desse modo, alterações no tamanho dos quelípodos ocasionam necessariamente mudanças nas interações intraespecíficas e do animal com o meio. Certamente, animais com quelípodos de tamanhos diferentes desempenham funções diferentes na população e no ambiente. Essas diferenças devem ocorrer em juvenis, fêmeas e machos de $M$. amazonicum. 

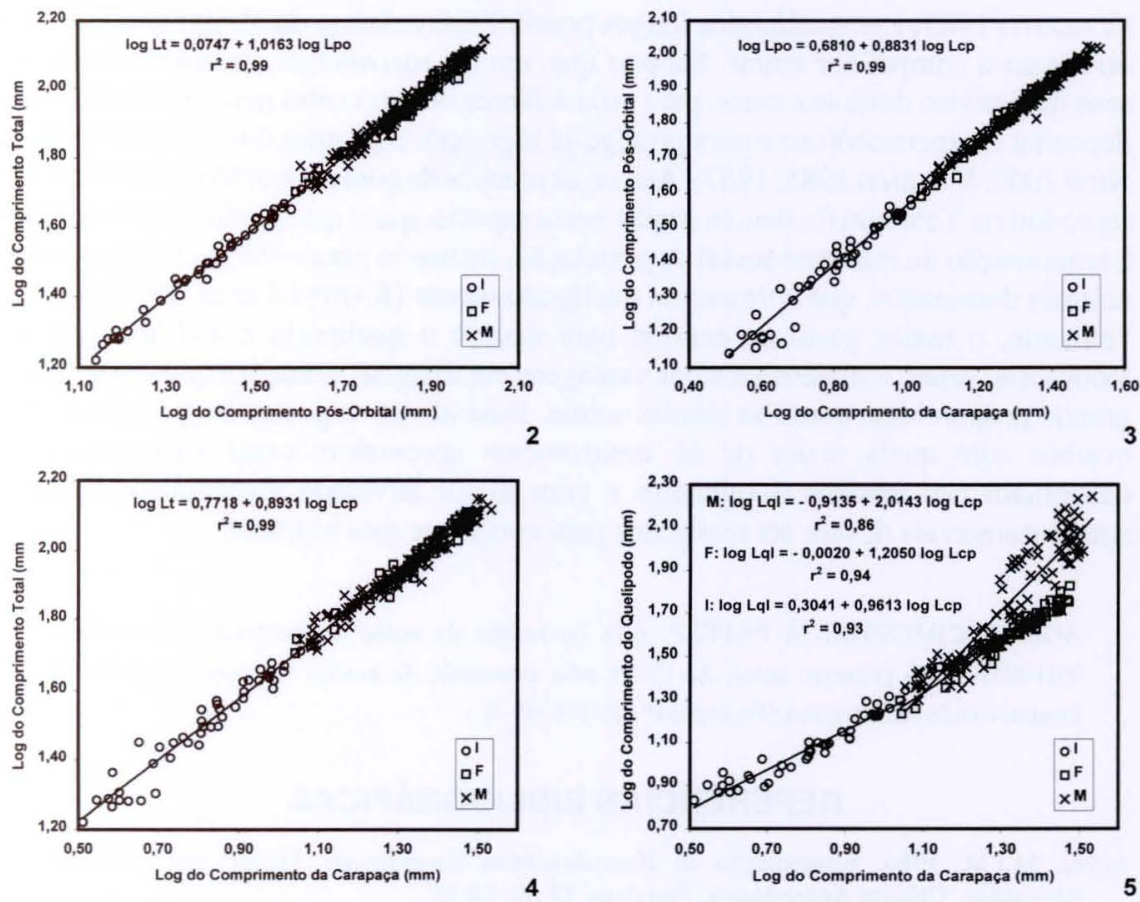

Figs 2-5. Relação entre: (2) comprimento total (Lt) e o comprimento pós-orbital (Lpo); (3) comprimento pós-orbital (Lpo) e o comprimento da carapaça (Lcp); (4) comprimento total (Lt) e o comprimento da carapaça (Lcp); (5) comprimento do quelípodo (Lql) e o comprimento da carapaça (LCp). (I) Indeterminados, (F) fêmeas, (M) machos.

Os machos maiores, que possuem os quelípodos muito desenvolvidos, deslocam muita energia e materiais para o crescimento e manutenção desse apêndice. Além disso, a quela muito grande diminui a capacidade de natação e até de locomoção sobre o substrato. Portanto, esses machos devem ser mais sedentários em relação aos animais jovens e fêmeas dessa espécie. Realmente, observou-se que esses dois últimos são mais ágeis que os machos mais desenvolvidos. Observou-se que as fêmeas de $M$. amazonicum apresentam elevada atividade gonadal durante quase todo o ano. Isto envolve grande gasto de energia e materiais para o desenvolvimento dos óvulos. Assim, as fêmeas canalizam a energia para a atividade reprodutiva. Esse processo poderia ocasionar um menor crescimento no quelípodo das fêmeas em relação ao dos machos.

O aumento no tamanho dos quelípodos ao longo do desenvolvimento dos machos deve conferir vantagens adaptativas que garantem a manutenção genética 
dessa característica na espécie. Os Macrobrachium têm hábito de viver entocados (VALENTI 1985) e os quelípodos longos possibilitam a defesa do abrigo sem sair dele ou deixar o competidor entrar. Sabe-se que, em $M$. rosenbergii, os machos utilizam seus quelípodos durante a corte, para virar a fêmea com o ventre para cima na hora de depositar o espermatóforo e para protegê-la logo após a postura dos ovos (ISMAEL \& NEW 2000; VALENTI 1985, 1987). Assim, os machos de quela maior têm maior sucesso reprodutivo. Também foi demonstrado, nessa espécie, que o quelípodo é essencial para a manutenção da estrutura social da população, inclusive para inibir o crescimento de animais dominados, que apresentam quelípodo menor (KARPLUS et al. 1992). Provavelmente, o maior gasto de energia para manter o quelípodo e a dificuldade de locomoção sejam compensados por vantagens em diversas situações que o quelípodo grande proporciona, como as citadas acima. Baseado no exposto, é esperado que os machos com quela maior de $M$. amazonicum apresentem essas vantagens e se constituam nos machos dominantes e com maior atividade reprodutiva. Estudos comportamentais devem ser realizados para confirmar esta hipótese.

AGRADECIMENTOS. À FAPESP, pela concessão da bolsa de mestrado (Processo $\mathrm{n}^{\text {o }}$ 99/10051-2), ao primeiro autor. Ao CNPq pela concessão do auxílio à pesquisa e Bolsa de Produtividade em Pesquisa (Processo nº 520748-96-4).

\section{REFERÊNCIAS BIBLIOGRÁFICAS}

Alves, M.I.M. 1986. Alimentação de Macrobrachium amazonicum (Heller) em condições de laboratório. Ciência Agronômica, Fortaleza, 17 (2): 19-23.

BarReto, A. \& A.M.C. SoAREs. 1982. Produção de pós-larvas de Macrobrachium amazonicum (Heller, 1862) (Decapoda, Palaemonidae), sob condições controladas de laboratório. Revta bras. Zool. 1 (1): 51-53.

Bialetzki, A.; K. Nakatani; G. Baumgartner \& G. Bond-Buckup. 1997. Occurrence of Macrobrachium amazonicum (Heller) (Decapoda, Palaemonidae) in Leopoldo's inlet (Ressaco do Leopoldo), upper Paraná river, Porto Rico, Paraná, Brazil. Revta bras. Zool. 14 (2): 379-390.

Bragagnoli, G. \& M. Grotta. 1995. Reprodução do camarão de água doce M. amazonicum (Heller, 1862) nos açudes públicos do nordeste brasileiro. Revista Nordestina de Biologia, Paraíba, 10 (2): 141-154.

Clayton, D.A. 1990. Crustacean allometric growth: a case for caution. Crustaceana, Leiding, 58 (3): 270-290.

GUEST, W.C. 1979. Laboratory life history of the palaemonid shrimp Macrobrachium amazonicum (Heller, 1862) (Decapoda, Palaemonidae). Crustaceana, Leiden, 37 (2): 141-152.

GURGEL, J.J.S. \& M.O.M. MATOS. 1984. Sobre a criação extensiva do camarão-canela, Macrobrachium amazonicum (Heller, 1862) nos açudes públicos do nordeste brasileiro. Anais Simpósio Brasileiro de Aqüicultura 3, São Carlos: 705.

Hartnoll, R.G. 1982. Growth, p. 111-96. In: D.E. Bliss (Ed.). The Biology of Crustacea. Embriology, Morphology and Genetics. New York, Academic Press, vol. 2, 440p.

Holthuis, L.B. 1952. A general revision of the Palaemonidae (Crustacea, Decapoda, Natantia) of the Americas. II. The subfamily Palaemonidae. Occasional Papers, Allan Hancock Foundation, Los Angeles, 12: 1-396.

Ismael, D. \& M.B. New. 2000. Biology, p. 18-40. In: M.B. NEw \& W.C. VAlenti (Eds). Freshwater Prawn Culture: The Farming of Macrobrachium rosenbergii. Londres, Blackwell Science, $\mathrm{XX}+443 \mathrm{p}$. 
Karplus, I.; G. Hulata; D. Ovadia \& R. JafFia. 1992. Social control of growth in Macrobrachium rosenbergii. IV. The role of claws in bull-runt interactions. Aquaculture, Amsterdam, 105: 281-296.

KURIS, A.M.; Z. RA'ANAN; A. SAGI \& D. COHEN. 1987. Morphotypic differentiation of male Malaysian giant prawns, Macrobrachium rosenbergii. Jour. Crustacean Biol., Laurence, 7: 219-237.

Kutty, M.N.; F. Herman \& H.L. Menn. 2000. Culture of other prawn species, p. 393-410. In: M.B. New \& W.C. Valenti (Eds). Freshwater Prawn Culture: The Farming of Macrobrachium rosenbergii. Londres, Blackwell Science, $\mathrm{XX}+443 \mathrm{p}$.

LoBĀO, V.L. \& F.B. LoNA. 1979. Biometria e isometria em Macrobrachium holthuisi (Genofre \& Lobão, 1976) (Crustacea, Decapoda). Bol. Inst. Pesca, São Paulo, 6: 17-50.

LoBÃo, V.L.; W.C. VALEnTI \& J.T.C. Melo. 1986. Crescimento relativo de Macrobrachium carcinus (L.) (Decapoda, Palaemonidae). Bol. Inst. Pesca, São Paulo, 13 (1): 105-109.

Lobão, V.L.; N.E.T. Rojas; H.P. Barros; M. Lace; M.T. Horikawa \& L.A.B.M. Lula. 1987. Determinação de densidades adequadas para a larvicultura de Macrobrachium amazonicum (Heller, 1862) (Crustacea, Decapoda, Palaemonidae). Bol. Inst. Pesca, São Paulo, 14: 45-49.

LoBÃo, V.L.; E.A. Roverso \& J.V. LomBARDI. 1994. Influência da densidade inicial de estocagem no desenvolvimento de Macrobrachium rosenbergii (De Man, 1879) e Macrobrachium amazonicum (Heller, 1862) (Decapoda, Palaemonidae) em laboratório. Bol. Inst. Pesca, São Paulo, 21: 11-17.

LOVETT, D.L. \& D.L. Felder. 1989. Application of regression techniques to studies of relative growth in crustaceans. Jour. Crustacean Biol., Laurence, 9 (4): 529-539.

Magalhães, C. 1985. Desenvolvimento larval obtido em laboratório de palemonídeos da região Amazônica: I. Macrobrachium amazonicum (Heller, 1862) (Crustacea, Decapoda). Amazoniana, Manaus, 9 (2): 247-274.

Mantelatto, F.L.M. \& A. Fransozo. 1994. Crescimento relativo e dimorfismo sexual em Hepatus pudinbundus (Herbst, 1785) (Decapoda, Brachyura) no litoral norte paulista. Papéis Avulsos Zool., São Paulo, 39 (4):33-48.

Moraes-Riodades, P.M.C. \& W.C. VAlENTI. 2001. Freshwater prawn farming in Brazilian Amazonia shows potential for economic and social development. Global Aquaculture Advocate, Saint Louis, 4 (5): 73-74.

Moraes-Riodades, P.M.C.; W.C. Valenti; A.S.L. Peralta \& M.D.L. Amorim. 1999. Carcinicultura de água doce no Estado do Pará: situação atual e perspectivas. Anais Congresso Brasileiro de Engenharia de Pesca, 9 e Congresso Latino-Americano de Engenharia de Pesca, 1, Recife: 598-604.

Moreira, L.C. \& O. OdinetZ-Collart. 1993. Migração vertical nictemeral das larvas do camarão Macrobrachium amazonicum num lago de várzea na Amazônia Central (Ilha do Careiro). Amazoniana, Manaus, 12 (3/4): 385-398.

Nagamine, C.M. \& A.W. Knight. 1980. Development, maturation, and function of some sexually dimorphic structures of malaysian prawn, Macrobrachium rosenbergii (De Man) (Decapoda, Palaemonidae) E.J. Brill. Crustaceana, Leiding, 39 (2): 141-152.

New, M.B.; S. Singholka \& M.N. KutTy. 2000. Prawn capture fisheries and enhancement, p. 411-428. In: M.B. New \& W.C. Valenti (Eds). Freshwater Prawn Culture: The Farming of Macrobrachium rosenbergii. Londres, Blackwell Science, $\mathrm{XX}+443 \mathrm{p}$.

Odinetz-Collart, O. 1987. La pêche crevettière de Macrobrachium amazonicum (Palaemonidae) dans le Bas-Tocantins après la fermeture du barrage de Tucurui. Rev. Hydrobiol. Trop. 20 (2): 131-144.

- 1991a. Stratégie de reproduction de Macrobrachium amazonicum en Amazonie Centrale. Crustaceana, Leiding, 61 (2): 253-270.

1991b. Tucurui dam and the populations of the prawn Macrobrachium amazonicum in the lower Tocantins (PA-Brasil): a four years study. Arch. Hidrobiol. 122 (2): 213-228.

Odinetz-Collart, O. \& L.C. Moreira. 1993. Potencial pesqueiro do camarão Macrobrachium amazonicum na Amazônia Central (Ilha do Careiro). Amazoniana, Manaus, 12 (3/4): 399-413.

Odinetz-Collart, O. \& C. Magalhães. 1994. Ecological constraints and life history strategies of

Revta bras. Zool. 19 (4): $1169-1176,2002$ 
Palaemonid prawns in Amazônia. Verh. Internat. Verein. Limnol., Stuttgart, 25: 2460-2467.

Odinetz-Collart, O. \& H. Rabelo. 1996. Variation in egg size of the fresh-water prawn Macrobrachium amazonicum (Decapoda: Palaemonidae). Jour. Crustacean Biol., Laurence, 16 (4): 684-688.

RAO, R.M. 1967. Studies on the biology of Macrobrachium rosenbergii (De Man) of the Hoogly estuary with notes on its fishery. Proc. Nat. Inst. Sci. Índia, Calcutta, 33 (5-6): 252-279.

Reigada, A.L.D. \& M.L. Negreiros-Fransozo. 1999. Maturidade Sexual em Hepatus pudibundus (Decapoda, Brachyura, Callapidae). Iheringia, Sér. Zool., Porto Alegre, 86: 159-164.

RiCKER, W.E. 1973. Linear regressions in fishery research. Jour. Fish. Res. Board Can., Ottawa, 30: 409-434.

Rodrigues, S.A. 1985. Sobre o crescimento relativo de Callichirus major (Say, 1918) (Crustacea, Decapoda, Thalassinidea). Bol. Zool. Univ. São Paulo 9: 195-211.

RoJAs, N.E.T.; V.L. LoBÃo \& H.P. BARRos. 1990. Métodos de manutenção de larvas de Macrobrachium amazonicum (Heller, 1862) (Crustacea, Decapoda, Palaemonidae). Bol. Inst. Pesca, São Paulo, 17: $15-26$.

RoMERO, M.E. 1982. Preliminary observations on potential of culture of Macrobrachium amazonicum in Venezuela, p. 411-416. In: M.B. New (Ed.) Giant Prawn Farming. Amsterdam, Elsevier, 532p.

Roverso, E.A.; V.L. LoBÃo \& M.T. HokriKawa. 1990. Arraçoamento intensivo de pós-larvas de Macrobrachium amazonicum (Heller, 1862) e Macrobrachium rosenbergii (De Man, 1868) (Decapoda, Palaemonidae) até a fase de juvenil. Bol. Inst. Pesca, São Paulo, 17: 91-98.

SCAICO, M.A. 1992. Fecundidade e fertilidade de Macrobrachium amazonicum (Crustacea, Decapoda) de um açude do Nordeste Brasileiro. Bol. Inst. Pesca, São Paulo, 19: 89-96.

SoKAL, R.R. \& F.J. RoHLF. 1995. Biometry. New York, Freeman, $3^{\mathrm{a}}$ ed., XIX+887p.

SPIVAK, E.D. \& C.C. BAS. 1999. First finding of the pelagic crab Planes marinus (Decapoda: Grapsidae) in southwestern Atlantic. Jour. Crustacean Biol., Laurence, 19(1): 72-76.

VAlEnTI, W.C. 1985. Cultivo de Camarões de Água Doce. São Paulo, Nobel, XII+82p.

- 1987. Comportamento reprodutivo de camarões de água doce, Anais Encontro Anual de Etologia 5, Jaboticabal: 195-202.

VALENTI, W.C.; V.L.LOBÃo \& J.T.C. MELLO. 1989. Crescimento relativo de Macrobrachium acanthurus (Wiegmann, 1836) (Crustacea, Decapoda, Palaemonidae). Revta bras. Zool. 6 (1): 1-8.

Volpato, G.L. \& K. Hoshino. 1984. Adaptative process derived from the agonitic behavior in the freswater prawn Macrobrachium iheringi (Ortmann, 1897). Bol. Fisiol. Animal, São Paulo, 8: 157-163.

Recebido em 10.VI.2002; aceito em 04.XI.2002 